\title{
LECTURES ON \\ QUANTUM MECHANICS
}

GORDON BAYM

Unitsersity of Illinois

\section{A II $\quad$ Advanced Bock Progrant}

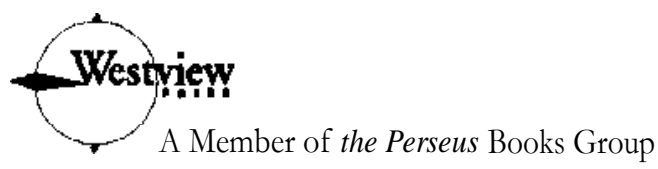




\section{CONTENTS}

Preface

Chapter 1 Photon Polarization • Transformation of Bases $\quad 8$

Angular Momenhun $\quad 13$

Amplitude Mechanics $\quad 20$

Unpolarized Light 25

Behavior of Photon Polarization in Matter 29

Problems 33

Chapter 2 Neutral K Mesons 38

Quantum Interference Effects $\quad 42$

Problems $\quad 45$

Chapter 3 The Motion of Particles in Quantum Mechanics 46

The Schrödinger Equation 46

State Vectors 53

Digression an the Delta Function $\quad 55$

Operators for Physical Quantities 59

The Free Particle $\quad 62$

Quantum Mechanical Motion as a Sum over Paths $\quad 69$

Particle in a Magnetic Field $\quad 74$

Aharonov-Bohm Effect $\quad 77$

$\begin{array}{ll}\text { Problems } & 79\end{array}$

Chapter 4 Potential Problems, Mostly in One Dimension 83

Properties of Hermitian Operators 83 
Energy Eigenstates $\quad 85$

One-Dimensional Barriers $\quad 88$

Tunneling $\quad 93$

Bound States $\quad 97$

Parity $\quad 99$

Transmission Resonances $\quad 104$

One-Dimensional Delta Function Potential 113

Periodic Potentials 116

The Harmonic Oscillator $\quad 123$

Problems 128

Chapter 5 Equations of Motion for Operators 134

Functions of Operators 134

Heisenberg Representation 136

Formal Solution for the Time Development Operator 142

Problems 145

Chapter 6 Orbital Angular Momentum and Central Potentials 148

Commutation Relations 148

Rotation $\quad 150$

Differential Operator Representation 153

Eigenfunctions and Eigenvalues $\quad 155$

Spherical Harmonics 159

Central Forces $\quad 162$

$\begin{array}{ll}\text { Problems } & 167\end{array}$

Chapter 7 The Hydrogen Atom $\quad 169$

Two Particle Systems 169

Hydrogen Atom Wave Functions 172

The Symmetry of the Hydrogen Atom 175

$\begin{array}{lr}\text { Problems } & 179\end{array}$

$\begin{array}{ll}\text { Chapter } 8 \text { Cooper Pairs } & 180\end{array}$

Problems 189

Chapter 9 Potential Scattering 191

Wave Packets 191

Cross Sections $\quad 196$

Partial Waves $\quad 197$

The Optical Theorem 201

The Born Approximation $\quad 202$

Properties of the Scattering Amplitude 204 
Low Energy Resonances $\quad 208$

Problems 212

Chapter 10 Coulomb Scattering 213

Analytic Properties of the Scattering Amplitude 217

Regge Poles 219

Problems 223

Chapter 11 Stationary State Perturbation Theory 225

The Wave Function Renormalization Constant 229

Degenerate Perturbation Theory 231

Van der Waals Interaction 234

Almost Degenerate Perturbation Theory 237

Brillouin-Wigner Perturbation Theory 241

Nonperturbative Methods $\quad 242$

Problems $\quad 244$

Chapter 12 Time-Dependent Perturbation Theory 246

First-Order Transitions: Golden Rule 248

Harmonic Perturbations 255

Second-Order Transitions $\quad 257$

Forward "Scattering»Amplitude 258

Problems 260

Chapter 13 Interaction of Radiation with Matter 262

Interaction Hamiltonian $\quad 264$

Absorption of Light $\quad 267$

Quantized Radiation Field 271

Einstein's A and B Coefficients 276

Details of Spontaneous Emission 278

Electric Dipole Transitions 281

Magnetic Dipole and Electric Quadrupole Transitions 287

Scattering of Light 289

Raman Scattering $\quad 295$

Problems 299

Chapter 14 Spin 1/2 302

Rotations in Spin Space $\quad 305$

Including Spatial Degrees of Freedom 308

Spin Magnetic Moment $\quad 310$

Precession $\quad 315$

Spin Resonance $\quad 317$ 
Motion in Inhomogeneous Magnetic Fields

Chapter 15 Addition of Angular Momenta

Addition of Two Angular Momenta 335

Clebsch-Cordan Technology

Scattering of Spin 1/2 Particles with Spinless Particles 342

Three Angular Momenta

Problems

Chapter 16 Isotopic Spin

Problems

Chapter 17 Rotations and Tensor Operators

Representations of Rotations

Tensor Operators

Multipole Radiation

Angular Momentum and the Harmonie Oscillator

Problems

Chapter 18 Identical Particles

Permutation and Symmetry

States of Noninteracting Identical Particles

Scattering of Identical Particles

Identity and Isotopic Spin

Problems

Chapter 19 Second Quantization

Creation and Annihilation Operators

Second Quantized Operators

Pair Correlation Functions

The Hanbury-Brown and Twiss Experiment

The Hamiltonian

Problems

Two-Electron Atoms

Hartree Approximation

Fermi-Thomas Approximation

Hartree-Fock

The Periodic Table

Splitting of Configurations 
Spin-Orbit Interaction

Spin-Orbit Splitting in Hartree-Fock

Zeeman Effect

Problems

Chapter 21 Molecules

Born-Oppenheimer Method

The 112-Ion

The Hydrogen Molecule

Pairing of Electrons

Spatially Directed Orbitals

Hybridization

Hydrocarbons

Chapter 22 Relativistic Spin Zero Particles:

Klein-Gordon Equation $\quad 499$

Negative Energy States and Antiparticles $\quad 504$

First-Order Klein--Cordon Equation $\quad 507$

Free Particle Wave Packets 513

Klein's Paradox $\quad 515$

Scattering by a Potential $\quad \mathbf{5 2 0}$

Bound State Problems 524

Nonrelativistic Limit $\quad 529$

Scalar Interactions $\quad 531$

Neutral Particles $\quad 532$

Problems $\quad 533$

Chapter 23 Relativistic Spin 1/2 Particles: Dirac Equation 534

Lorentz Transformation of Spin $\quad 534$

Dirac Equation $\quad 543$

Free Particle Solutions $\quad 548$

Currents $\quad 552$

Nonrelativistic Limit $\quad 558$

Second-Order Dirac Equation $\quad 563$

Dirac Hydrogen Atom 564

Hyperfine Structure $\quad 572$

The Lamb Shift $\quad 574$

Dirac Hole Theory $\quad 580$

Problems $\quad 586$

Index 